\title{
A comparison of the efficacy and safety of once-daily fluticasone furoate/vilanterol with twice-daily fluticasone propionate/ salmeterol in moderate to very severe COPD
}

\author{
Alvar Agustí1,2, Luis de Teresa ${ }^{3}$, Wilfried De Backer ${ }^{4}$, Michael T. Zvarich $^{5}$, \\ Nicholas Locantore ${ }^{5}$, Neil Barnes ${ }^{6}$, Jean Bourbeau ${ }^{7}$ and Courtney Crim ${ }^{5}$
}

\begin{abstract}
Affiliations: ${ }^{1}$ Thorax Institute, Hospital Clinic, IDIBAPS, University of Barcelona, Barcelona, ${ }^{2} \mathrm{FISIB}, \mathrm{CIBER}$ Enfermedades Respiratorias (CIBERES), Mallorca, and ${ }^{3}$ Medicina Innovadora, Clinica Mediterranea de Neurociencias, Alicante, Spain. 4 University Hospital, University of Antwerp, Antwerp, Belgium. ${ }^{5}$ GlaxoSmithKline, Research Triangle Park, NC, USA. ${ }^{6}$ Respiratory Medicine, The London Chest Hospital, Barts Health NHS Trust, London, UK. ${ }^{7}$ Respiratory Epidemiology and Clinical Research Unit (RECRU), McGill University, Montreal, Quebec, Canada.
\end{abstract}

Correspondence: A. Agustí, Institut del Tòrax, Hospital Clínic, Villarroel 170, Escala 3, Planta 5, 08036 Barcelona, Spain. E-mail: alvar.agustiđclinic.ub.es

ABSTRACT Fluticasone furoate/vilanterol trifenatate (FF/VI) is a once-daily inhaled corticosteroid/longacting $\beta_{2}$-agonist combination in development for chronic obstructive pulmonary disease (COPD) treatment. We compared the efficacy and safety of FF/VI versus fluticasone propionate/salmeterol (FP/SAL) twice daily over 12 weeks.

Moderate to very severe COPD patients received FF/VI 100/25 $\mu \mathrm{g}$ once daily in the morning $(\mathrm{n}=266)$ or FP/SAL 500/50 $\mu \mathrm{g}$ twice daily $(\mathrm{n}=262)$. The primary end-point was a change from baseline in $0-24 \mathrm{~h}$ weighted mean forced expiratory volume in $1 \mathrm{~s}(\mathrm{wmFEV} 1)$ at 12 weeks. Additional end-points included time to $100 \mathrm{~mL}$ improvement from baseline on day 1 and a change from baseline in St George's Respiratory Questionnaire (SGRQ). Safety was also assessed.

wmFEV1 (mean $130 \mathrm{~mL}$ ) was greater and time to $100 \mathrm{~mL}$ improvement shorter (median $16 \mathrm{~min}$ ) with FF/VI than FP/SAL (weighted mean $108 \mathrm{~mL}$, median $28 \mathrm{~min}$ ). Health status (SGRQ total score) improved in both groups (FF/VI -4.3 units, FP/SAL -3.0 units). Differences between treatments were not statistically significant. Six patients in the FF/VI (2\%) and three in the FP/SAL (1\%) arm experienced serious adverse events, none of which were considered to be drug related.

Improvements in lung function and health status were not significantly different between FF/VI 100/ $25 \mu \mathrm{g}$ once daily and FP/SAL 500/50 $\mu \mathrm{g}$ twice daily; there was no apparent difference between the safety profiles of either therapy.

@ERSpublications

Once-daily fluticasone furoate/vilanterol trifenatate and twice-daily fluticasone propionate/ salmeterol act similarly http://ow.ly/sdj0x

This article has supplementary material available from www.erj.ersjournals.com

Received: March 262013 | Accepted after revision: July 302013 | First published online: Oct 102013

Clinical trial: This study is registered at www.clinicaltrials.gov with identifier number NCT01342913; GlaxoSmithKline Study Code HZC113107.

Support statement: This work was funded by GlaxoSmithKline. Editorial support from G. Weller (Gardiner-Caldwell Communications, Macclesfield, UK) was also funded by GlaxoSmithKline.

Conflict of interest: Disclosures can be found alongside the online version of this article at www.erj.ersjournals.com 


\section{Introduction}

In patients with moderate to very severe chronic obstructive pulmonary disease (COPD), combined treatment with an inhaled corticosteroid (ICS) and a long-acting $\beta_{2}$ agonist (LABA) improves lung function [1] and health status [2], reduces the annual exacerbation rate [3] and slows disease progression as assessed by the rate of decline of forced expiratory volume in $1 \mathrm{~s}$ (FEV1) [4]. Currently available ICS/LABA combinations require twice-daily dosing (e.g. fluticasone propionate (FP) and salmeterol (SAL)).

Fluticasone furoate (FF)/vilanterol trifenatate (VI) is a novel ICS/LABA combination being developed for once-daily administration, a dosing scheme that may improve adherence [5]. This is potentially important, as low therapeutic compliance is associated with poor clinical outcomes in COPD $[6,7]$. FF is structurally distinct from FP and has enhanced glucocorticoid receptor binding [8]. In vitro, VI exhibits $\geqslant 1000$-fold selectivity for the $\beta_{2}$ receptor over the $\beta_{1}$ or $\beta_{3}$ receptors [9]. Furthermore VI is an ante-drug [10], which degrades to metabolites with reduced/negligible potency upon entry to the systemic circulation. In vitro, the main metabolites of VI are $\geqslant 2500$-fold less potent than VI at the $\beta_{2}$ receptor [11].

Phase IIb dose-ranging trials of VI in COPD [12] and FF in asthma [13-15] have shown that both therapies are efficacious and exhibit a once-daily dosing profile [16, 17]. FF/VI has been studied at strengths of 50/ $25 \mu \mathrm{g}, 100 / 25 \mu \mathrm{g}$ and 200/25 $\mu \mathrm{g}$ in a small 4-week crossover study [18], and in larger 24-week lung function studies [19,20] and 52-week exacerbation studies [21]; none of these studies compared FF/VI with an established ICS/LABA combination, such as FP/SAL. The current study is the first to compare head-to-head the efficacy and safety of FF/VI (100/25 $\mu$ g once daily) versus FP/SAL (500/50 $\mu$ g twice daily), conducted over 12 weeks of treatment in patients with moderate to very severe COPD.

\section{Methods}

\section{Patients and ethics}

We studied male and female adults aged $\geqslant 40$ years, with a smoking history of $\geqslant 10$ pack-years and a postbronchodilator (salbutamol) FEV1/forced vital capacity ratio of $\leqslant 0.70$ and a FEV1 $\leqslant 70 \%$ predicted [22]. Patients had to have experienced at least one moderate COPD exacerbation (requiring treatment with oral corticosteroid/antibiotic) or severe exacerbation (leading to hospitalisation) within the past 3 years. Patients with a current diagnosis of asthma, serious underlying disease or infections, hospitalisation due to COPD within 12 weeks of screening, or acute worsening of COPD (defined as use of corticosteroids or antibiotics) within 6 weeks of screening were not included. All patients signed their informed consent, and the protocol was approved by local ethics review committees of the 61 centres where the study was conducted. The study was conducted in accordance with the Declaration of Helsinki and Good Clinical Practice guidelines.

\section{Study design}

This was a 12-week, randomised, multicentre (61 centres in Europe and Asia), double-blind, doubledummy, parallel-group, comparative efficacy/safety study. The study is registered at www.clinicaltrials.gov (identifier number NCT01342913); GlaxoSmithKline study number HZC113107.

Following screening, patients entered a 2 -week, placebo run-in period, after which they were randomised in a 1:1 ratio to receive in a double-blind manner FF/VI 100/25 $\mu \mathrm{g}$ once daily in the morning via the ELLIPTA dry powder inhaler (GlaxoSmithKline, Ware, UK), which emits a dose of $92 \mu \mathrm{g}$ FF and $22 \mu \mathrm{g}$ VI, or FP/SAL $500 / 50 \mu \mathrm{g}$ twice daily via the Accuhaler (GlaxoSmithKline, Uxbridge, UK), for 12 weeks. Patients receiving FF/VI also took a placebo via Accuhaler once in the morning and once in the evening, and patients receiving FP/SAL took a placebo once in the morning using the ELLIPTA dry powder inhaler. Patient randomisation schedule was computer generated by GlaxoSmithKline, and treatments were assigned via a telephone call to the interactive voice response system using RandAll and RAMOS (both GlaxoSmithKline, London, UK). To account for a potential confounding effect of bronchial reversibility, randomisation was stratified based upon the patient's reversibility, defined as a change in FEV1 of $\geqslant 12 \%$ and $\geqslant 200 \mathrm{~mL} 10-15$ min after four inhalations of salbutamol. Reversibility was assessed at the screening visit, prior to the 2-week placebo runin period. For standardisation across all subjects, study-supplied salbutamol was used and administered via a study-supplied valved holding chamber (AeroChamber Plus; GlaxoSmithKline) for the purpose of reversibility testing. Compliance with treatment was assessed by reviewing the dose counters on both inhalers at randomisation (day 1), day 28, day 56 and on day 84. Salbutamol was supplied to patients for symptomatic relief during the study. Ipratropium, mucolytics and oxygen for $\leqslant 12 \mathrm{~h}$ per day were permitted provided their dose did not change during the study. Likewise, cardioselective $\beta$-blockers, intranasal sodium cromoglycate or nedocromil sodium, intranasal corticosteroids, diuretics and medications for other disorders were also permitted, provided the dose remained constant wherever possible and their use was not expected to affect lung function. Medications not permitted during the course of the study are provided in the online supplementary material. 


\section{Efficacy assessment}

The primary efficacy end-point of the study was the 24-h effect of FF/VI on lung function after 12 weeks of treatment (day 84), as compared with FP/SAL. This was assessed through the change from baseline in weighted mean (wm) FEV1. Baseline FEV1 was defined as the mean of two FEV1 assessments taken 30 min and 5 min pre-dose on day 1 of the study (i.e. after the 2 -week placebo run-in period). Day 84 FEV 1 was defined as the weighted mean of $15 \mathrm{FEV} 1$ assessments taken at 5, 15, 30 and $60 \mathrm{~min}$ pre-dose, and at 2, 4, 6, 8, 12, 13, 14, 16, 20 and $24 \mathrm{~h}$ post-dose. All FEV1 assessments were conducted using standardised equipment that met or exceeded the suggested standards [23]. All sites were issued with Biomedical Systems Vitalograph 6800 Fleisch pneumotach (Biomedical Systems (BMS), Brussels, Belgium) for spirometry assessments prior to study start, and received training from BMS. ECG data was also obtained from BMS programming and equipment. Each site electronically transmitted both the spirometry and ECG data to BMS, where the data underwent quality control and best test review by the over-readers who were certified by BMS to analyse both the pulmonary function and ECG data.

Secondary efficacy end-points were: 1) time to $100 \mathrm{~mL}$ increase from baseline from $0-4 \mathrm{~h}$ on day 1 ; and 2) change from baseline in trough FEV1 on day 85, i.e. the comparison of the FEV1 recorded 24 h post-dose on day 84 with the baseline measure.

Other efficacy end-points included changes in health status, as determined by St George's Respiratory Questionnaire (SGRQ) for COPD and rescue-free $24 \mathrm{~h}$ periods. Health status was assessed on day 1 (i.e. following the 2-week placebo run-in) and day 84 of the study, or at the point of early withdrawal if prior to day 84 . Finally, a post hoc analysis of the difference in lung function $0-4 \mathrm{~h}, 0-12 \mathrm{~h}$ and $12-24 \mathrm{~h}$ post-dose on day 84 was also conducted.

\section{Safety assessment}

Safety and tolerability were assessed by the incidence of adverse events and severe adverse events, coded using the Medical Dictionary for Regulatory Activities. Adverse events of special interest were also assessed. These comprised adverse events known to be associated with ICS and/or LABA therapy and included bone disorders, cardiovascular effects, effects on potassium, effects on glucose, hypersensitivity, local steroid effects, ocular effects, pneumonia, lower respiratory tract infections excluding pneumonia, systemic steroid effects and tremors. The incidence of COPD exacerbations and pneumonias, as well as abnormalities of oropharyngeal examinations, clinical chemistry and haematology assessments, vital signs and ECG measurements, were also recorded.

\section{Statistical analysis}

We assumed a treatment difference (FF/VI-FP/SAL) of $60 \mathrm{~mL}$ for $24 \mathrm{~h}$ wmFEV1 with a standard deviation of $190 \mathrm{~mL}$. The $60 \mathrm{~mL}$ treatment difference in wmFEV1 on which the study was powered was based on extrapolation of previous wmFEV1 results with SAL [1] and VI [12] taking into account the putative minimal clinically important difference for trough FEV1 [24, 25]. Based upon this analysis, a $60 \mathrm{~mL}$ treatment difference in wmFEV1 was determined to be an appropriate difference for superiority of one active treatment compared to another (for further explanation please see online supplementary material). Accordingly, we calculated that a sample size of 212 evaluable patients in each arm of the study was needed to detect a statistically significant difference at a $5 \%$ level (two-sided) with $90 \%$ power. Assuming a withdrawal rate of $15 \%$, we aimed to randomise a minimum of 250 patients per treatment arm.

Data are shown as mean \pm SD or proportion, as appropriate. The two treatment arms were compared using ANCOVA, with efficacy results displayed as mean \pm SD. To account for multiplicity across primary and secondary efficacy end-points, a p-value $<0.05$ was required for the primary end-point to allow statistical significance to be inferred for secondary end-points. For this reason a p-value is presented only for the primary comparison; otherwise, point estimates and 95\% confidence intervals are presented, but no formal statistical comparison can be done. All efficacy and safety measurements were analysed in the intent-to-treat (ITT) population, as defined by all patients who were randomised to treatment and who received at least one dose of study medication.

\section{Results}

\section{Patient characterisation}

Figure 1 presents the consort diagram of the study. A total of 528 patients were randomised and comprised the ITT population. 243 (91\%) patients in the FF/VI arm and $246(94 \%)$ in the FP/SAL arm completed the study. The demographic and clinical characteristics of participants (ITT population), including age, sex, ethnicity, body mass index, comorbidities, medications received and mean FEV1 were similar at baseline regardless of study arm (table 1$)$. 


\section{Efficacy: primary end-point}

An improvement from baseline in $0-24 \mathrm{~h}$ wmFEV1 on day 84 was observed with both FF/VI (mean \pm SD $130 \pm 222 \mathrm{~mL})$ and FP/SAL $(108 \pm 221 \mathrm{~mL})$; the difference in improvement between the two arms $(22 \mathrm{~mL})$ did not reach statistical significance $(\mathrm{p}=0.282)$. A post hoc comparison of changes in wmFEV 1 between arms did show a difference from $0-4 \mathrm{~h}$ and $0-12 \mathrm{~h}$ post-dose (daytime), but not from 12-24 h post-dose (table 2 and fig. 2).

\section{Efficacy: secondary end-points}

As explained in the methods section, because statistical significance was not achieved for the primary endpoint, the analysis plan established that statistical significance could not be inferred for comparisons of secondary efficacy end-points. Hence, all differences described below should be considered as descriptive only.

The mean change from baseline in trough FEV1 on day 85 (an indicator of 24-h effect) was $111 \mathrm{~mL}$ in the FF/VI and $88 \mathrm{~mL}$ in the FP/SAL arms (ITT population) with a mean treatment difference of $23 \mathrm{~mL}$ (95\% CI -20-66) (table 2). The median time to reach an increase in FEV1 of $\geqslant 100 \mathrm{~mL}$ on day 1 was 16 min in the FF/VI treatment group and $28 \mathrm{~min}$ in the FP/SAL treatment group. The minimal clinically important difference [26] for change from baseline in SGRQ was achieved in the FF/VI group (mean \pm SD $-4.3 \pm 11.8$ ) but not in the FP/SAL group $(-3.0 \pm 11.8)$ (table 2). The proportion of rescue-free 24-h periods was similar between treatments $(61.6 \%$ for FF/VI and $58.5 \%$ for FP/SAL during the first week of the study, and $62.5 \%$ for $\mathrm{FF} / \mathrm{VI}$ and $59.8 \%$ for FP/SAL over the entire duration of the study).

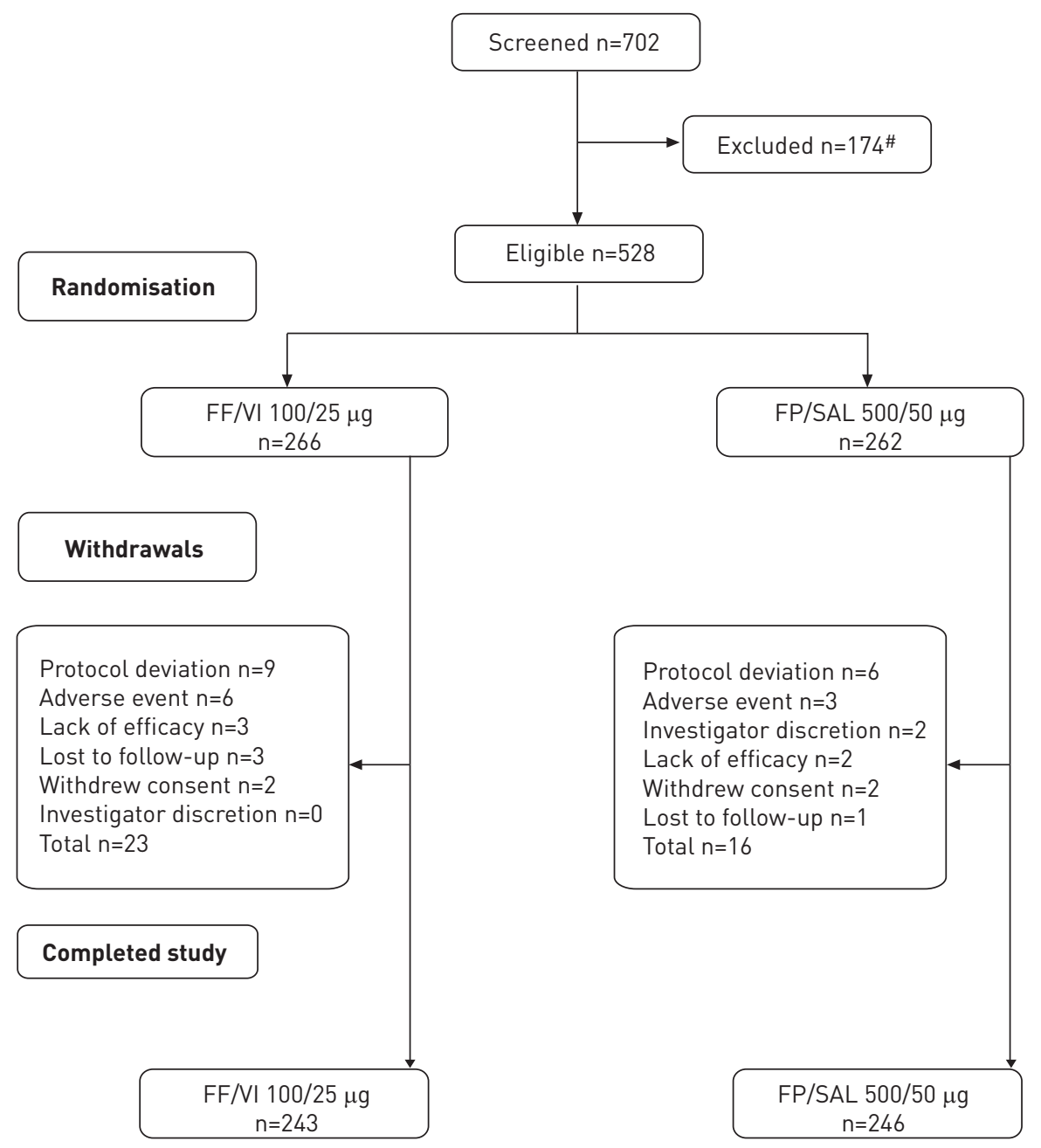

FIGURE 1 Consort diagram of the study. FF: fluticasone furoate; FP: fluticasone propionate; SAL: salmeterol; VI: vilanterol trifenatate. ${ }^{\#}$ : reasons for exclusion comprised: did not meet inclusion/met exclusion criteria $(n=66)$, did not meet continuation criteria $(n=89)$, withdrew consent $(n=7)$, investgator discretion $(n=6)$, protocol deviation $(n=5)$ and adverse event $(n=2)$. Subjects could be excluded from randomisation for more than one reason. 
FF/VI $100 / 25 \mu g$

\section{6}

$63.0 \pm 8.1$

$212(80)$

$218(82)$

48 (18)

0

$26.2 \pm 5.7$

Body mass index $\mathbf{~} \mathbf{g} \cdot \mathbf{m}^{-2}$

Commonly reported prior COPD therapy $\%$

Tiotropium

SAL

FP

Formoterol

Ipratropium

\section{Comorbid conditions}

Vascular disorders

Metabolism and nutrition disorders

Cardiac disorders

\section{Lung function}

Screening post-bronchodilator FEV1 L Screening post-bronchodilator FEV1 \% pred

Screening post-bronchodilator FEV1/FVC

Screening FEV1 reversibility \%

Screening reversible

Baseline pre-bronchodilator FEV1 L

Baseline pre-bronchodilator FEV1 \% pred

Baseline pre-bronchodilator FVC L

Baseline pre-bronchodilator FEV1/FVC \%

Baseline SGRQ total score

COPD exacerbations

Requiring antibiotics/corticosteroids in prior 3 years ${ }^{+}$

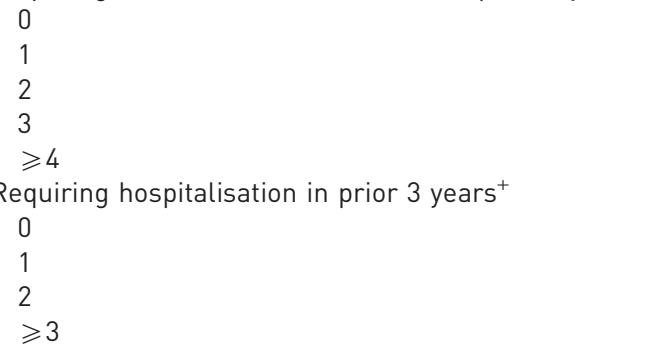

FP/SAL $500 / 50 \mu g$

262

$62.9 \pm 9.1$

$221(84)$

208 (79)

$53(20)$

$1(<1)$

$25.6 \pm 4.9$

$$
\begin{gathered}
112(42) \\
54(20) \\
37(14) \\
\\
1.43(0.43) \\
47.94(11.50) \\
0.49(0.10) \\
11.7 \pm 11.9 \\
74(28) \\
1.28 \pm 0.43 \\
43.0 \pm 11.9 \\
2.77 \pm 0.78 \\
46.6(9.5) \\
46.7 \pm 17.0
\end{gathered}
$$

$125(48)$

$63(24)$

$25(10)$

$1.44(0.46)$

47.64 (11.91)

$0.48(0.10)$

$11.9 \pm 11.8$

73 (28)

$1.30 \pm 0.45$

$43.0+12.3$

$2.83 \pm 0.80$

$46.0(10.2)$

$44.8+17.8$

$\begin{array}{cc}29(11) & 29(11) \\ 125(47) & 126(48) \\ 52(20) & 52(20) \\ 32(12) & 36(14) \\ 28(11) & 19(7) \\ & \\ 200(75) & 190(73) \\ 53(20) & 60(23) \\ 12(5) & 11(4) \\ 1(<1) & 1(<1)\end{array}$

Data are presented as $n$, mean \pm SD or $n(\%)$, unless otherwise stated. FF: fluticasone furoate; VI: vilanterol trifenatate; FP: fluticasone propionate; SAL: salmeterol; COPD: chronic obstructive pulmonary disease; FEV1: forced expiratory volume in 1 s; FVC: forced vital capacity; SGRQ: St George's Respiratory Questionnaire. " : some subjects may have received more than one therapy, therapy stopped within 3 months of screening visit; ${ }^{\top}$ : defined as $\geqslant 12 \%$ and $\geqslant 200 \mathrm{~mL}$ post-salbutamol; ${ }^{+}$: percentages do not sum to 100 due to rounding.

\section{Safety}

The occurrence of on-treatment adverse events and drug-related adverse events was similar between the two study arms (table 3). More adverse events leading to withdrawal and more on-treatment severe adverse events were observed with FF/VI than with FP/SAL (table 3). Headache and nasopharyngitis were the most frequently reported on-treatment adverse events. The most frequently reported drug-related adverse event was oral candidiasis (preferred term) $(\mathrm{FF} / \mathrm{VI}, \mathrm{n}=2$ and FP/SAL, $\mathrm{n}=4)$. Two out of the three adverse events leading to withdrawal in the FP/SAL arm were due to pneumonia (versus one event in the FF/VI arm), and three out of the six adverse events leading to withdrawal in the FF/VI arm were due to cardiac disorders (versus none in the FP/SAL arm), specifically atrial fibrillation $(n=2)$ and congestive heart failure $(n=1)$. None of the adverse events leading to withdrawal and none of the on-treatment severe adverse events were considered by the study investigators to be treatment related. There were no fatal on-treatment adverse 
TABLE 2 Efficacy assessments

FF/VI 100/25 $\mu \mathrm{g}$

$\begin{array}{cc}266 & 262 \\ 130 \pm 222 & 108 \pm 221 \\ 111 \pm 241 & 88 \pm 241 \\ -4.3 \pm 11.8 & -3.0 \pm 11.8 \\ 205 \pm 226 & 162 \pm 227 \\ 175 \pm 225 & 128 \pm 225 \\ 88 \pm 229 & 87 \pm 229\end{array}$

FP/SAL $500 / 50 \mu g$
Least squares mean difference $(95 \% \mathrm{CI})$

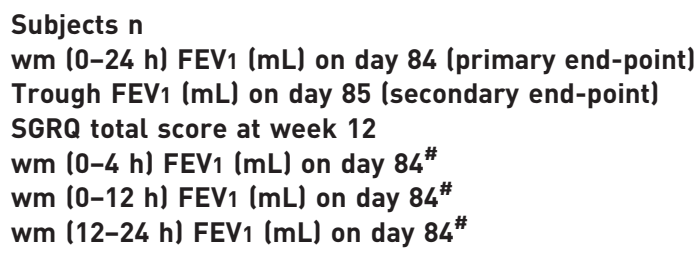

$22(-18-63)^{\circ}$

$23(-20-66)$

$-1.3(-3.5-0.8)$

$43(2-83)$

$46(5-88)$

$1(-41-43)$

Data are presented as mean \pm SD, unless otherwise stated. FF: fluticasone furoate; VI: vilanterol trifenatate; FP: fluticasone propionate; SAL: salmeterol; wm: weighted mean; FEV1: forced expiratory volume in $1 \mathrm{~s}$; SGRQ: St George's Respiratory Questionnaire. "\#: post hoc analyses; ^: $p=0.282$.

events; one fatal event during the post-treatment follow-up period of FF/VI treatment (congestive heart failure) was not considered to be treatment related.

The occurrence of adverse events of special interest was generally low and similar across the two treatment groups, although more patients experienced cardiovascular adverse events in the FF/VI group (nine versus one) and more patients experienced local steroid effects in the FP/SAL group (10 versus three). Of the local steroid effects, three events each of oral candidiasis and oropharyngeal candidiasis, and one event of dysphonia were considered to be drug-related in the FP/SAL group, while two events of oral candidiasis and one event of dysphonia were considered to be drug-related in the FF/VI group (table 3). COPD exacerbations occurred in six (2\%) patients receiving FF/VI and seven (3\%) patients receiving FP/SAL. All but two of these patients were withdrawn from the study and all exacerbations resolved satisfactorily.

Three patients experienced four pneumonia events during the study (one from the FF/VI group and two from the FP/SAL group); one subject in the FP/SAL group experienced two pneumonias prior to being withdrawn. The protocol required that patients be withdrawn in the event of pneumonia; this subject should have been withdrawn at their first pneumonia but was not and so was considered a protocol violator. No abnormalities in laboratory values of potential clinical concern were observed during this study. There was no significant difference in pulse rate between treatment groups, either at randomisation or after 12 weeks of treatment (data not shown).

\section{Discussion}

This study shows that the efficacy and safety of the novel once-daily combination of FF/VI 100/25 $\mu \mathrm{g}$ in patients with moderate to very severe COPD over 12 weeks is not significantly different to that of the currently available FP/SAL 500/50 $\mu \mathrm{g}$ twice daily dose.

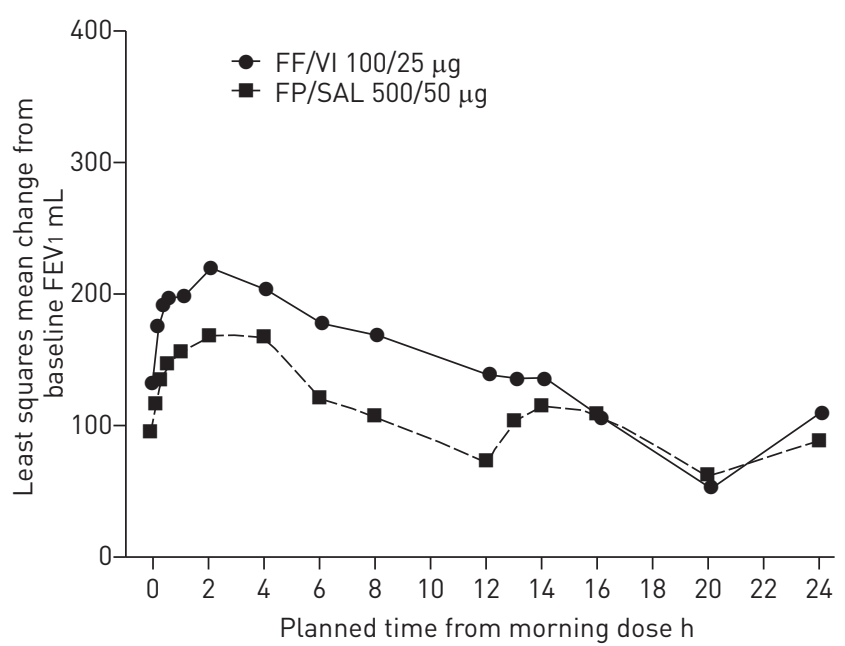

FIGURE 2 Change from baseline in the least squares mean forced expiratory volume in $1 \mathrm{~s}$ (FEV1) on day 84 in the intent-to-treat population. FF: fluticasone furoate; VI: vilanterol trifenatate; FP: fluticasone propionate; SAL: salmeterol. 
TABLE 3 On-treatment adverse events and events of special interest by treatment arm (intent-to-treat population)

FF/VI $100 / 25 \mu \mathrm{g}$

\begin{tabular}{|c|c|}
\hline 266 & 262 \\
\hline 73 (27) & $68(26)$ \\
\hline $6(2)$ & $3(1)$ \\
\hline $6(2)$ & $3(1)$ \\
\hline $4(2)$ & 9 (3) \\
\hline $9(3)^{\oplus}$ & $1(<1)$ \\
\hline $2(<1)$ & $1(<1)$ \\
\hline $2(<1)$ & 0 \\
\hline $2(<1)$ & 0 \\
\hline $1(<1)$ & 0 \\
\hline $1(<1)$ & 0 \\
\hline $1(<1)$ & 0 \\
\hline $1(<1)$ & 0 \\
\hline $3(1)$ & $10(4)^{5}$ \\
\hline $2(<1)^{+}$ & $4(2)^{f}$ \\
\hline 0 & $3(1)^{+}$ \\
\hline 0 & $3(1)$ \\
\hline $1(<1)^{+}$ & $1(<1)^{+}$ \\
\hline $2(<1)$ & $2(<1)$ \\
\hline $2(<1)$ & 0 \\
\hline $1(<1)$ & $2(<1)$ \\
\hline $1(<1)$ & $1(<1)$ \\
\hline $1(<1)^{+}$ & 0 \\
\hline 0 & $2(<1)^{\# \#}$ \\
\hline 0 & $1(<1)$ \\
\hline $20(8)$ & $18(7)$ \\
\hline $8(3)$ & $12(5)$ \\
\hline $10(4)$ & $3(1)$ \\
\hline $3(1)$ & 7 (3) \\
\hline $2(<1)$ & 4 (2) \\
\hline
\end{tabular}

Subjects $\mathbf{n}$

Summary

Any on-treatment adverse events

Adverse events leading to permanent discontinuation from the study or withdrawal of study treatment

Any on-treatment severe adverse events

Drug-related adverse events ${ }^{\#}$

\section{Adverse events of special interest (special interest term)}

Cardiovascular effects

Hypertension

Atrial fibrillation

Tachycardia

Angina pectoris

Bradycardia

Coronary artery disease

Oedema peripheral

Local steroid effects

Oral candidiasis

Oropharyngeal candidiasis

Oropharyngeal pain

Dysphonia

Hypersensitivity

LRTI excluding pneumonia

Pneumonia

Ocular effects

Tremor

Effects on glucose

Bone disorders

On-treatment adverse events occurring in $\geqslant 2 \%$ of patients in any treatment group

Headache

Nasopharyngitis

Back pain

Cough

Oral candidiasis
FP/SAL $500 / 50 \mu \mathrm{g}$

262

$8(26)$

(1)

(3)

0

$(4)^{5}$

$(2)^{+}$

$3(1)$

$(<1)^{+}$

$(<1)$

$1<1$

1) \#\#

$(<1)$

$18(7)$

$2(5)$

$7(3)$

$4(2)$

Data are presented as n (\%). FF: fluticasone furoate; VI: vilanterol trifenatate; FP: fluticasone propionate; SAL: salmeterol; LRTI: lower respiratory tract infection. ${ }^{\#}$ : as determined by the investigator; ${ }^{\text {"}}$ : one subject had angina pectoris and coronary artery disease; ${ }^{+}$: all events were considered drug-related; ${ }^{\S}$ : one subject had dysphonia and oral candidiasis; ${ }^{f}$ : three out of the four events were considered drug-related; ${ }^{\# \#}$ : one out of of the two events was considered drug-related.

An ICS/LABA combination is a well-established therapeutic strategy for patients with COPD [27]. The improvements from baseline in lung function over 12 weeks with FF/VI and FP/SAL both exceeded $100 \mathrm{~mL}$, which is regarded as a clinically perceptible difference [24, 25], although the $22 \mathrm{~mL}$ difference between the two treatment arms was not statistically significant. However, two observations (fig. 2) deserve comment. First, the FEV1 profile in the early part of the day appeared enhanced with FF/VI. This observation merits confirmation in other studies, as it is possible that it could translate into additional clinical benefits, such as occurrence of COPD exacerbations [28]. Secondly, the expected second peak in the FEV1 area under the curve observed after the administration of FP/SAL (fig. 2) did not surpass that of FF/VI, probably reflecting the pharmacological potency of FF/VI.

As recently emphasised by the Global Strategy for the Diagnosis, Management and Prevention of Chronic Obstructive Pulmonary Disease 2011 document, the assessment and treatment of symptoms is an important component of COPD management [27]. The SGRQ for COPD is a well-established instrument for the assessment of health status in patients with COPD [29]. A difference of $\geqslant 4$ points in the SGRQ total score versus placebo at study end, or $\geqslant 4$ points from baseline is considered to be the minimal clinically important difference for this measure [26]. We observed that treatment with FF/VI over 12 weeks demonstrated an improvement of $\geqslant 4$ units (mean change 4.3), while FP/SAL achieved an improvement of $<4$ units (mean change 3.0). This supports a positive effect of FF/VI on patient's health status, but as the comparison is the 
change from baseline as opposed to a difference from placebo in change from baseline (there was no placebo arm in the current study), the results must be interpreted in light of this. Indeed, a placebo effect on health status has previously been noted in other interventional clinical trials in COPD [30, 31], possibly as a consequence of the overall health benefits of the regular clinic visits associated with participation in a clinical trial.

To further compare the 24-h efficacy profile of the two treatment arms, we performed a post hoc analysis of wmFEV 1 at $0-4 \mathrm{~h}, 0-12 \mathrm{~h}$ and $12-24$ h post-dose on day 84 . We observed a larger wmFEV1 with FF/VI than with FP/SAL in both early periods, i.e. during the daytime. This may theoretically contribute to improved exercise tolerance and health-status, but requires further prospective assessment. During the second $12 \mathrm{~h}$ period on day 84 there was a $1-\mathrm{mL}$ difference in wmFEV1 between FF/VI $(88 \mathrm{~mL})$ and FP/SAL $(87 \mathrm{~mL})$. It is important to recall that the $88 \mathrm{~mL}$ change from baseline recorded with FF/VI during this period occurred without the need for a second dose of therapy. As with any putative daytime effects, the potential impact of lung function changes upon night-time symptoms in COPD is unclear [32] and requires additional prospective assessment.

FF/VI differs from other currently available ICS/LABA combinations in that it is taken once daily whereas others require twice-daily dosing [33]. This simplified dosing regimen may improve adherence [34, 35], and consequently has the potential to provide better disease control and improved outcomes [36]. However, the present study was not designed to assess adherence to therapy and any relationship it may have to outcome. Indeed, mean adherence in both study arms, as assessed by evaluation of inhaler dose counters, was $97.5 \%$, which suggests that the results observed herein reflect those achieved with optimal adherence. Whether such adherence would be observed in a real-world setting, where adherence to COPD therapy is known to be much lower [37], and whether dosing frequency would impact adherence and potentially outcome, remains to be established, ideally in the setting of an effectiveness study.

The rates of adverse events and severe adverse events were similar in both study arms; however, there were differences between the study arms in terms of adverse events of specific interest. 1) More local effects of steroids were observed in the FP/SAL arm than in the FF/VI arm. This may potentially be due to lower daily ICS exposure with FF/VI $\left(100 \mu \mathrm{g} \cdot \mathrm{day}^{-1}\right)$ than with FP/SAL $\left(1000 \mu \mathrm{g} \cdot \mathrm{day}^{-1}\right)$, although it must be considered that budesonide equivalent values for FF have not yet been determined (in asthma the daily budesonide dose equivalent to $1000 \mu \mathrm{g}$ of FP is $1600 \mu \mathrm{g}$ ) [38]. 2) More cardiovascular effects were observed in the FF/ VI arm than in the FP/SAL arm. Overall, LABA-mediated effects, such as those on potassium and glucose, or tremors, occurred infrequently with either treatment, and there was little difference in their incidence between the study arms. In addition, two 6-month trials with FF/VI 100/25 $\mu \mathrm{g}$ did not show an increase in cardiovascular adverse events compared with placebo $[19,20]$.

Our study did have some limitations. The absence of a placebo arm limited the interpretation of data for all end-points. Also, the relatively short duration of 12 weeks limited our ability to draw firm conclusions with respect to exacerbations, pneumonia and other systemic adverse events, as longer follow-up is normally required for differences in the incidence of these events to become apparent. Furthermore, there was no attempt to document potential pneumonia events radiologically. It is possible, therefore, that a larger sample size and/or an unselected COPD population may have identified any rare adverse events that were not observed in our relatively small and restricted population.

In conclusion, FF/VI (100/25 $\mu \mathrm{g}$ once daily) and FP/SAL (500/50 $\mu \mathrm{g}$ twice daily) both produced improvements from baseline in lung function and health status, in patients with moderate to very severe COPD; there was no statistical difference between the improvements observed with FP/SAL and those observed with FF/VI. The safety profile of each treatment did not markedly differ in terms of adverse events and severe adverse events, though more local steroid effects were observed with FP/SAL and more cardiovascular effects were observed with FF/VI. These findings suggest that FF/VI 100/25 $\mu$ g provides an improvement in lung function and health status that is not superior to, but is comparable to, that provided by FP/SAL $500 / 50 \mu \mathrm{g}$, and furthermore is provided by once-daily as opposed to twice-daily therapy.

\section{Acknowledgements}

The authors thank participants for their willingness to contribute to medical research and field investigators for their dedication and quality of their work.

Editorial support in the form of development of an outline and first draft under the guidance of the lead author, copyediting of drafts of this manuscript and generation of tables and figures was conducted by Geoff Weller (GardinerCaldwell Communications, Macclesfield, UK). 


\section{References}

1 Mahler DA, Wire P, Horstman D, et al. Effectiveness of fluticasone propionate and salmeterol combination delivered via the Diskus device in the treatment of chronic obstructive pulmonary disease. Am J Respir Crit Care Med 2002; 166: 1084-1091.

2 Calverley PM, Anderson JA, Celli B, et al. Salmeterol and fluticasone propionate and survival in chronic obstructive pulmonary disease. N Engl J Med 2007; 356: 775-789.

3 Kardos P, Wencker M, Glaab $\mathrm{T}$, et al. Impact of salmeterol/fluticasone propionate versus salmeterol on exacerbations in severe chronic obstructive pulmonary disease. Am J Respir Crit Care Med 2007; 175: 144-149.

4 Celli BR, Thomas NE, Anderson JA, et al. Effect of pharmacotherapy on rate of decline of lung function in chronic obstructive pulmonary disease: results from the TORCH study. Am J Respir Crit Care Med 2008; 178: 332-338.

5 Price D, Robertson A, Bullen K, et al. Improved adherence with once-daily versus twice-daily dosing of mometasone furoate administered via a dry powder inhaler: a randomized open-label study. BMC Pulm Med 2010; 10: 1.

6 Restrepo RD, Alvarez MT, Wittnebel LD, et al. Medication adherence issues in patients treated for COPD. Int J Chron Obstruct Pulmon Dis 2008; 3: 371-384.

7 Vestbo J, Anderson JA, Calverley PM, et al. Adherence to inhaled therapy, mortality and hospital admission in COPD. Thorax 2009; 64: 939-943.

8 Biggadike K, Bledsoe RK, Hassell AM, et al. X-ray crystal structure of the novel enhanced-affinity glucocorticoid agonist fluticasone furoate in the glucocorticoid receptor-ligand binding domain. J Med Chem 2008; 51: 3349-3352.

9 Slack RJ, Barrett VJ, Morrisson VS, et al. In vitro pharmacological characterization of vilanterol, a novel long-acting $\beta_{2}$-adrenoceptor agonist with 24-hour duration of action. J Pharmacol Exp Ther 2013; 344: 218-230.

10 Procopiou PA, Barrett VJ, Bevan NJ, et al. Synthesis and structure-activity relationships of long-acting $\beta_{2}$ adrenergic receptor agonists incorporating metabolic inactivation: an antedrug approach. J Med Chem 2010; 53: $4522-4530$.

11 Harrell AW, Siederer SK, Bal J, et al. Metabolism and disposition of vilanterol, a long-acting $\beta_{2}$-adrenoceptor agonist for inhalation use in humans. Drug Metab Dispos 2013; 41: 89-100.

12 Hanania NA, Feldman G, Zachgo W, et al. The efficacy and safety of the novel long-acting $\beta_{2}$ agonist vilanterol in patients with COPD: a randomized placebo-controlled trial. Chest 2012; 142: 119-127.

13 Bateman ED, Bleecker ER, Lötvall J, et al. Dose effect of once-daily fluticasone furoate in persistent asthma: a randomized trial. Respir Med 2012; 106: 642-650.

14 Bleecker ER, Bateman ED, Busse WW, et al. Consistently favorable safety profile of fluticasone furoate (FF), a oncedaily (OD) inhaled corticosteroid (ICS), across a range of treatment steps in patients with uncontrolled asthma. Am J Respir Crit Care Med 2011; 183: A1300.

15 Busse WW, Bleecker ER, Bateman ED, et al. Fluticasone furoate demonstrates efficacy in patients with asthma symptomatic on medium doses of inhaled corticosteroid therapy: an 8-week, randomised, placebo-controlled trial. Thorax 2011; 67: 35-41.

16 Woodcock A, Bleecker ER, Busse WW, et al. Fluticasone furoate: once-daily evening treatment versus twice-daily treatment in moderate asthma. Respir Res 2011; 12: 160

17 Sterling R, Lim J, Frith L, et al. Efficacy and optimal dosing interval of the long-acting $\beta_{2}$ agonist, vilanterol, in persistent asthma: a randomised trial. Respir Med 2012; 106: 1110-1115.

18 Boscia JA, Pudi KK, Zvarich MT, et al. Effect of once-daily fluticasone furoate/vilanterol on 24-hour pulmonary function in patients with chronic obstructive pulmonary disease: a randomized, three-way, incomplete block, crossover study. Clin Ther 2012; 34: 1655-1666.

19 Kerwin EM, Scott-Wilson C, Sanford L, et al. A randomised trial of fluticasone furoate/vilanterol (50/25 $\mu$ g; 100/ $25 \mu \mathrm{g}$ ) on lung function in COPD. Respir Med 2013; 107: 560-569.

20 Martinez FJ, Boscia J, Feldman G, et al. Fluticasone furoate/vilanterol (100/25; 200/25 $\mu \mathrm{g})$ improves lung function in COPD: a randomised trial. Respir Med 2013; 107: 550-559.

21 Dransfield MT, Bourbeau B, Jones PW, et al. Once-daily inhaled fluticasone furoate and vilanterol versus vilanterol only for prevention of exacerbations of COPD: two replicate double-blind, parallel-group, randomised controlled trials. Lancet Respir Med 2013; 1: 210-223.

22 Celli BR, MacNee W ATS/ERS Task Force. Standards for the diagnosis and treatment of patients with COPD: a summary of the ATS/ERS position paper. Eur Respir J 2004; 23: 932-946.

23 Miller MR, Hankinson J, Brusasco V, et al. Standardisation of spirometry. Eur Respir J 2005; 26: 319-338.

24 Cazzola M, MacNee W, Martinez FJ, et al. Outcomes for COPD pharmacological trials: from lung function to biomarkers. Eur Respir J 2008; 31: 416-469.

25 Donohue JF. Minimal clinically important differences in COPD lung function. COPD 2005; 2: 111-124.

26 Jones PW. St. George's Respiratory Questionnaire: MCID. COPD 2005; 2: 75-79.

27 Vestbo J, Hurd SS, Agustí AG, et al. Global strategy for the diagnosis, management, and prevention of chronic obstructive pulmonary disease: GOLD executive summary. Am J Respir Crit Care Med 2013; 187: 347-365.

28 Anzueto A. Impact of exacerbations on COPD. Eur Respir Rev 2010; 19: 113-118.

29 Meguro M, Barley EA, Spencer S, et al. Development and validation of an improved, COPD-specific version of the St. George Respiratory Questionnaire. Chest 2007; 132: 456-463.

30 Chapman KR, Rennard SI, Dogra A, et al. Long-term safety and efficacy of indacaterol, a long-acting $\beta_{2}$-agonist, in patients with COPD: a randomized, placebo-controlled study. Chest 2011; 140: 68-75.

31 Tashkin DP, Doherty DE, Kerwin E, et al. Efficacy and safety characteristics of mometasone furoate/formoterol fumarate fixed-dose combination in patients with moderate to very severe COPD: findings from pooled analysis of two randomized, 52-week placebo-controlled trials. Int J Chron Obstruct Pulmon Dis 2012; 7: 73-86.

32 Agusti A, Hedner J, Marin JM, et al. Night-time symptoms: a forgotten dimension of COPD. Eur Respir Rev 2011; 20: 183-194.

33 Calverley P, Pauwels R, Vestbo J, et al. Combined salmeterol and fluticasone in the treatment of chronic obstructive pulmonary disease: a randomized controlled trial. Lancet 2003; 361: 449-456.

34 Claxton AJ, Cramer J, Pierce C. A systematic review of the associations between dose regimens and medication compliance. Clin Ther 2001; 23: 1296-1310. 
35 Breekveldt-Postma NS, Penning-van Beest FJ, Siiskonen SJ, et al. The effect of discontinuation of antihypertensives on the risk of acute myocardial infarction and stroke. Curr Med Res Opin 2008; 24: 121-127.

Bourbeau J, Bartlett SJ. Patient adherence in COPD. Thorax 2008; 63: 831-838.

37 Lareau SC, Yawn BP. Improving adherence with inhaler therapy in COPD. Int J Chron Obstruct Pulmon Dis 2010; 5: 401-406.

38 Global Initiative for Asthma. Global Strategy for Asthma Management and Prevention, 2012. www.ginasthma.org/ documents/4 Date last updated: December 2012. Date last accessed: January 30, 2013. 